\title{
Prevalence of human Papillomavirus in head and neck cancer in Mexico: a case-control study
} Samantha Carrera-Cáceres*1,2, Dulce M Hernández-Hernández³, Teresa Apresa ${ }^{3}$, José F Gallegos-Hernández ${ }^{3}$, Miriam Guido-Jiménez ${ }^{2,4}$ and Alejandro García-Carrancá2,4

\author{
Address: ${ }^{1}$ Facultad de Química, UNAM, Ciudad Universitaria, México City, México, ${ }^{2}$ Division of Research, Instituto Nacional de Cancerología \\ (INCan), México City, México, ${ }^{3}$ Medical Research Unit in Oncology Diseases, Pathology Department, Oncology Hospital, Centro Medico Siglo \\ XXI, Instituto Mexicano del Seguro Social (IMSS), México City, México and ${ }^{4}$ Instituto de Investigaciones Biomédicas, UNAM, Ciudad Universitaria, \\ México City, México \\ Email: Samantha Carrera-Cáceres* - sam_carrerac@yahoo.com \\ * Corresponding author
}

from 24th Annual Meeting of the National Cancer Institute of Mexico

Mexico City, Mexico. 14-17 February 2007

Published: 5 February 2007

BMC Cancer 2007, 7(SuppI I):AI5 doi:I0.II86/I47I-2407-7-SI-AI5

This article is available from: http://www.biomedcentral.com/I47I-2407/7/SI/AI5

(C) 2007 Carrera-Cáceres et al; licensee BioMed Central Ltd.

\section{Background}

Smoking or chewing tobacco and alcohol consumption are the major risk factors for development of Head and Neck Squamous Cell Carcinoma (HNSCC). However, studies suggest that a subset of these cancers could be associated with HPV infections. This association is reinforced by the fact that the most prevalent HPV types found in cervical carcinoma are those most frequently detected in Head and Neck Cancer. The prevalence of HPV in this type of cancer is highly variable with detection rates varying from 0 to $100 \%$ depending on the study and the anatomic location of the neoplasm. Few studies have been made in Mexican populations to determine prevalence of HPV in HNSCC. The aim of this study is to determine the prevalence of HPV in samples of patients with HNSCC and healthy individuals in a Mexican Population and to establish the types of HPV and HPV16 variants present in these samples.

\section{Materials and methods}

We conducted a case-control study in 100 samples of HNSCC and 100 control subjects. DNA from samples of exfoliated cells and biopsies was extracted using the salting out method, amplification of $\beta$-globin gene by PCR was used to asses DNA quality. HPV presence was evaluated by PCR with MY09/MY11 and GP5+/GP6+ primers.
DNA sequencing was used to determine HPV types and variant types.

\section{Results}

So far we had evaluated 50 samples of case patients, 46 were $\beta$-globin positive. HPV was detected in $72 \%(33 / 46)$ of the samples; $61.5 \%$ of carcinomas of the oral cavity, $74 \%$ of larynx and $100 \%$ of oropharynx. HPV type 16 was the most prevalent one (47\%). Some variants of HPV type 16 have been determined and European (E) variants had been the most frequent ones.

\section{Conclusion}

HPV DNA was found in a high percentage of HNSCCs. HPV may preferentially infect certain anatomic sites of the Head and Neck because prevalence was greater in the samples of the oropharynx than any other site, although analysis of a greater number of samples is needed to confirm these observations. As previously documented for Cervical Cancer, the most frequent type found was HPV type 16 and the variant type was European (E). 